\title{
Dual role of YAP: oncoprotein and tumor suppressor
}

\author{
Eekhoon Jho \\ Department of Life Science, University of Seoul, Seoul, Republic of Korea \\ Correspondence to: Eekhoon Jho, PhD. Department of Life Science, University of Seoul, Seoul 02504, Republic of Korea. Email: ej70@uos.ac.kr. \\ Provenance: This is an invited Editorial commissioned by the Section Editor Chunlin Ou (Cancer Research Institute of Central South University, \\ Changsha, China). \\ Comment on: Huang H, Zhang W, Pan Y, et al. YAP Suppresses Lung Squamous Cell Carcinoma Progression via Deregulation of the DNp63-GPX2 \\ Axis and ROS Accumulation. Cancer Res 2017;77:5769-81.
}

Submitted Aug 13, 2018. Accepted for publication Oct 15, 2018.

doi: $10.21037 /$ jtd.2018.10.70

View this article at: http://dx.doi.org/10.21037/jtd.2018.10.70

YAP (Yes-associated protein) is a terminal mediator of the Hippo signaling pathway. Hippo signaling is known as a key regulator of organ size by its ability to control cell proliferation and apoptosis $(1,2)$. The Hippo gene was named after the mutant eye overgrowth phenotype in Drosophila which resembles the shape of a hippopotamus $(3,4)$. Extensive genetic screening in Drosophila has led to the identification of upstream and downstream regulators of the Hippo signaling pathway including Merlin-Hippo-WartsYorkie $(1,2)$. Notably, all orthologs of Hippo pathway exist in metazoan: Nf2-Mst1/2-Lats1/2-YAP, respectively $(1,2)$. Tissue-specific deletion of $N f 2, M s t 1 / 2$ or Lats1/2 leads to hyperplasia, organ overgrowth, and finally tumorigenesis, suggesting their role as tumor suppressors (5-7). However, liver-specific expression of YAP results in hepatomegaly and tumors, indicating that YAP is an oncoprotein (8). Hippo signaling controls tissue homeostasis and organ growth by relaying a signaling cascade. First, the activation of Nf2 by various upstream signals derived from cell contact, energy stress, or GPCR signaling enhances the phosphorylation of Mst $1 / 2$ and Lats $1 / 2$ kinases. This leads to the phosphorylation of YAP by Lats1/2; the phosphorylated YAP is retained in the cytoplasm via the interaction with 14-3-3 and is eventually down-regulated by b-TrCPmediated proteasomal degradation $(1,2)$. However, in the absence of signals for Mst1/2 and Lats1/2 kinase, the unphosphorylated form of YAP enters the nucleus. Nuclear YAP interacts with the transcription factor TEAD and enhances the expression of genes involved in anti-apoptosis and proliferation $(1,2)$.

The current available data report high levels of nuclear
YAP with poor prognosis in the liver and various types of human cancer, for example non-small lung cancer (NSCLC) $(9,10)$, gastric cancer $(11,12)$, and colorectal cancer (CRC) $(13,14)$, suggesting that YAP is an oncoprotein (Table 1). Thus, lowering the level and activity of YAP has been considered as a direct strategy to inhibit cancer progression; yet, the issue is not that simple. Previous reports propose that YAP can behave as a tumor suppressor in different cellular contexts (Table 1). It was shown that YAP interacts with p73 tumor suppressor protein, a homologue of p53, in the nuclei and enhances the expression of pro-apoptotic genes such as $\$ 53 A I P 1$ and $B A X$ in response to DNA damage $(15,16)$. Similarly, RASSF1A, a tumor suppressor whose expression is reduced in various human cancers (21), increases the expression of pro-apoptotic gene puma via promoting the interaction between YAP and p73 in the nuclei to induce apoptosis (17). Consistent with its role as a tumor suppressor, the level of YAP was decreased or lost in breast cancers and its knockdown in breast cancer cell lines suppressed anoikis and increased migration and invasiveness although it was not examined whether these phenotypes were p73-dependent (18). Another way by which YAP acts as a tumor suppressor is switching its binding partner from TEAD to RUNX3 (19). Ectopic expression of RUNX3 in MKN28 gastric cancer cells reduced tumorigenicity which requires the interaction between YAP and RUNX3 (19).

In addition, Huang et al. reported a new mechanism in which YAP functions as a tumor suppressor in a recent issue of Cancer Research (20). Initially, the authors screened a chemical library with the objective of selecting small molecules with significant effects on the cell death of 
Table 1 Dual roles of YAP

Supporting evidences for YAP as an oncoprotein

Tissue-specific deletion of Nf2, Mst1/2 or Lats1/2, which results in increased YAP, leads to hyperplasia, organ overgrowth, and finally tumorigenesis (5-7)

Liver-specific expression of YAP results in hepatomegaly and tumors (8)

High levels of nuclear YAP with poor prognosis in the liver and various types of human cancer (9-14)

Supporting evidences for YAP as a tumor suppressor

YAP interacts with p73 and enhances the expression of pro-apoptotic genes in response to DNA damage $(15,16)$

Enhanced interaction between YAP and p73 by RASSF1A increases the expression of pro-apoptotic gene puma (17)

The level of YAP was decreased or lost in breast cancers and its knockdown in breast cancer cell lines suppressed anoikis and increased migration and invasiveness (18)

YAP switches binding partner from TEAD to RUNX3. Enhanced interaction between YAP and RUNX3 reduced tumorigenicity of MKN28 gastric cancer cells (19)

The accumulation of ROS due to the reduced expression of DNp63 and GPX2 by YAP caused cell death in lung squamous cell carcinoma cell lines (20)

YAP, Yes-associated protein.

human lung squamous cell carcinoma (SCC) cell line, L78. The screening allowed the authors to identify two molecules sharing similar chemical structures as their top hits: digitoxin and digoxin. Digitoxin is a cardiac glycoside which is widely used in clinic for treating heart failure (22). Since digitoxin is known to inhibit the interaction between the WW domain of YAP and canonical PPxY ligands (23), the authors examined the cytoplasmic localization of YAP under digitoxin treatment. Treatment of digitoxin reduced the level of YAP phosphorylation at S127, a site known to be phosphorylated by Lats1/2 kinases, and induced the nuclear localization of YAP. While it is likely that these phenotypes were due to the inhibition of the interaction between Lats1/2 and YAP by digitoxin, the authors did not examine this possibility. Knockdown of either MST1 or LATS1, which results in an increased level of nuclear YAP, attenuated lung SCC cell proliferation and colony formation in a YAPTEAD-dependent manner. These results suggest that YAP indeed acts as a tumor suppressor in lung SCC cells. Gene expression microarray profiling and gene set enrichment analysis (GSEA) after ectopic expression of YAP in lung SCC cells exhibited that YAP activation promoted ROS accumulation via reducing the expression ROS scavenging genes. Among these, the level of GPX2, a gene that encodes the antioxidant enzyme glutathione peroxidase, was most significantly reduced. The authors previously reported that YAP overexpression reduces the level $\mathrm{DNp} 63$, which is an isoform of $\mathrm{p} 63$, a member of the $\mathrm{p} 53$ family, by upregulating the transcriptional repressor ZEB2 (24). Interestingly, GPX2 was known as a direct target of DNp63 (25). Based on previous reports along with additional experiments, the authors claim that the accumulation of ROS due to the reduced expression of DNp63 and GPX2 by YAP caused cell death in lung SCC cells. However, whether the level of ZEB2 is increased by digitoxin was not examined. A clinically significant finding was that digitoxin treatment inhibits the growth of lung SCC with a low level of YAP in patient-derived xenograft (PDX) model, whose results provide a therapeutic strategy for the treatment of lung SCC patients with low levels of YAP.

In conclusion, YAP can act as either an oncoprotein or a tumor suppressor depending on the cellular context. In recent review by $\mathrm{Ou} e t \mathrm{al}$. also comprehensively analyzed the oncogenic and tumor suppressor functions of YAP in the differential status of colorectal cancer progression (26). This leads to following question: What are the specific cellular contexts required for the differential role of YAP? There are multiple possibilities. First, epigenetic differences during embryonic development may control different YAP target gene expression. YAP is involved in fate determination of mouse trophectoderm from the inner cell mass during early embryonic development by enhancing the expression of the transcription factor $C d x 2$ (27). However, despite the increased YAP level, the level of Cdx2 was low in colorectal cancer (28). These data suggest that different epigenetic modification of the $C d x 2$ locus may respond to YAP- 
TEAD distinctively in tumor and embryonic environment. Second, the relative availability of YAP binding partners in the nuclei may determine the differential role of YAP. As described above, exchanging binding partners from TEAD to RUNX3 can shift the role of YAP from oncoprotein to tumor suppressor. Recently, Lin et al. showed that environmental stress promotes the cytoplasmic translocation of TEAD, which selectively suppresses the oncogenic activity of YAP (29). Although it has not been examined in human tumors, it will be interesting to test whether different cellular localization of TEAD in various cancer cells may determine the response to the increase in YAP levels. Third, the degree of Hippo signaling may be different depending on the cellular context. Cai et al. reported that while YAP is dispensable for the homeostasis of normal intestine, YAP is essential for the dextran sodium sulfate (DSS)-induced intestinal regeneration (30). However, when Sav1, an upstream negative regulator of YAP, was deleted, colonic polyps were induced, and this crypt hyperplasia was suppressed by the loss of YAP. These data indicate that YAP activity is inhibited by the activation of upstream Hippo signaling components in normal intestine, whereas the activity YAP should be increased during the DSS-induced intestinal regeneration by reducing upstream Hippo activity. Overall, the activity of Hippo signaling may be different in different cellular context.

Generally, it is assumed that YAP functions as an oncoprotein due to its potent role in regulating the expression of genes specifically involved in cell proliferation and anti-apoptosis. However, recent reports suggest that in addition to its oncogenic role, YAP may act as a tumor suppressor depending on cellular contexts. This may seem surreal at first but increasing amount of experimental evidence clearly pinpoints that YAP has tumor suppressor activities. Thus, therapeutic efforts to diminish YAP levels in various types of tumors including lung cancer without the consideration of cellular contexts requires the need for caution, since the therapeutic treatment may result in increased tumor-growth instead. Taking into consideration the differential role of YAP in different cellular contexts would make a better approach in overcoming tumor progression.

\section{Acknowledgements}

Funding: This research was supported by the National Research Foundation of Korea (NRF) Grant funded by the Korean Government (MSIP) (NRF-
2016R1E1A1A01943544 and NRF-2016R1A5A1010764) to E Jho.

\section{Footnote}

Conflicts of Interest: The author has no conflicts of interest to declare.

\section{References}

1. Kim W, Jho EH. The history and regulatory mechanism of the Hippo pathway. BMB Rep 2018;51:106-18.

2. Meng Z, Moroishi T, Guan KL. Mechanisms of Hippo pathway regulation. Genes Dev 2016;30:1-17.

3. Harvey KF, Pfleger CM, Hariharan IK. The Drosophila Mst ortholog, hippo, restricts growth and cell proliferation and promotes apoptosis. Cell 2003;114:457-67.

4. Wu S, Huang J, Dong J, et al. hippo encodes a Ste-20 family protein kinase that restricts cell proliferation and promotes apoptosis in conjunction with salvador and warts. Cell 2003;114:445-56.

5. Zhang N, Bai H, David KK, et al. The Merlin/NF2 tumor suppressor functions through the YAP oncoprotein to regulate tissue homeostasis in mammals. Dev Cell 2010;19:27-38.

6. Lu L, Li Y, Kim SM, et al. Hippo signaling is a potent in vivo growth and tumor suppressor pathway in the mammalian liver. Proc Natl Acad Sci U S A 2010;107:1437-42.

7. St John MA, Tao W, Fei X, et al. Mice deficient of Lats1 develop soft-tissue sarcomas, ovarian tumours and pituitary dysfunction. Nat Genet 1999;21:182-6.

8. Dong J, Feldmann G, Huang J, et al. Elucidation of a universal size-control mechanism in Drosophila and mammals. Cell 2007;130:1120-33.

9. Guo J, Wu Y, Yang L, et al. Repression of YAP by NCTD disrupts NSCLC progression. Oncotarget 2017;8:2307-19.

10. Chaib I, Karachaliou N, Pilotto S, et al. Co-activation of STAT3 and YES-Associated Protein 1 (YAP1) Pathway in EGFR-Mutant NSCLC. J Natl Cancer Inst 2017;109(9).

11. Zhou Z, Zhu JS, Gao CP, et al. siRNA targeting YAP gene inhibits gastric carcinoma growth and tumor metastasis in SCID mice. Oncol Lett 2016;11:2806-14.

12. Ma LG, Bian SB, Cui JX, et al. LKB1 inhibits the proliferation of gastric cancer cells by suppressing the nuclear translocation of Yap and beta-catenin. Int J Mol Med 2016;37:1039-48. 
13. Huang YJ, Yang CK, Wei PL, et al. Ovatodiolide suppresses colon tumorigenesis and prevents polarization of M2 tumor-associated macrophages through YAP oncogenic pathways. J Hematol Oncol 2017;10:60.

14. Ling HH, Kuo CC, Lin BX, et al. Elevation of YAP promotes the epithelial-mesenchymal transition and tumor aggressiveness in colorectal cancer. Exp Cell Res 2017;350:218-25.

15. Strano S, Monti O, Pediconi N, et al. The transcriptional coactivator Yes-associated protein drives p73 genetarget specificity in response to DNA Damage. Mol Cell 2005; 18:447-59.

16. Strano S, Munarriz E, Rossi M, et al. Physical interaction with Yes-associated protein enhances p73 transcriptional activity. J Biol Chem 2001;276:15164-73.

17. Matallanas D, Romano D, Yee K, et al. RASSF1A elicits apoptosis through an MST2 pathway directing proapoptotic transcription by the p73 tumor suppressor protein. Mol Cell 2007;27:962-75.

18. Yuan M, Tomlinson V, Lara R, et al. Yes-associated protein (YAP) functions as a tumor suppressor in breast. Cell Death Differ 2008;15:1752-9.

19. Jang JW, Kim MK, Lee YS, et al. RAC-LATS1/2 signaling regulates YAP activity by switching between the YAPbinding partners TEAD4 and RUNX3. Oncogene 2017;36:999-1011.

20. Huang H, Zhang W, Pan Y, et al. YAP Suppresses Lung Squamous Cell Carcinoma Progression via Deregulation of the DNp63-GPX2 Axis and ROS Accumulation. Cancer Res 2017;77:5769-81.

21. Agathanggelou A, Cooper WN, Latif F. Role of the Rasassociation domain family 1 tumor suppressor gene in

Cite this article as: Jho E. Dual role of YAP: oncoprotein and tumor suppressor. J Thorac Dis 2018;10(Suppl 33):S3895S3898. doi: $10.21037 /$ jtd.2018.10.70 human cancers. Cancer Res 2005;65:3497-508.

22. Menger L, Vacchelli E, Kepp O, et al. Trial watch: Cardiac glycosides and cancer therapy. Oncoimmunology 2013;2:e23082.

23. Sudol M, Shields DC, Farooq A. Structures of YAP protein domains reveal promising targets for development of new cancer drugs. Semin Cell Dev Biol 2012;23:827-33.

24. Gao Y, Zhang $W$, Han X, et al. YAP inhibits squamous transdifferentiation of Lkb1-deficient lung adenocarcinoma through ZEB2-dependent DNp63 repression. Nat Commun 2014;5:4629.

25. Yan W, Chen X. GPX2, a direct target of $\mathrm{p} 63$, inhibits oxidative stress-induced apoptosis in a p53-dependent manner. J Biol Chem 2006;281:7856-62.

26. Ou C, Sun Z, Li S, et al. Dual roles of yes-associated protein (YAP) in colorectal cancer. Oncotarget 2017;8:75727-41.

27. Nishioka N, Inoue K, Adachi K, et al. The Hippo signaling pathway components Lats and Yap pattern Tead4 activity to distinguish mouse trophectoderm from inner cell mass. Dev Cell 2009;16:398-410.

28. Liang K, Zhou G, Zhang Q, et al. Expression of hippo pathway in colorectal cancer. Saudi J Gastroenterol 2014;20:188-94.

29. Lin KC, Moroishi T, Meng Z, et al. Regulation of Hippo pathway transcription factor TEAD by p38 MAPK-induced cytoplasmic translocation. Nat Cell Biol 2017;19:996-1002.

30. Cai J, Zhang N, Zheng Y, et al. The Hippo signaling pathway restricts the oncogenic potential of an intestinal regeneration program. Genes Dev 2010;24:2383-8. 